\title{
Research on the Development of the Recycle
}

\section{Treatment Industry of the Municipal Solid}

\section{Wastes under the Background of China's Urbanization}

\author{
Yongzhuan Su \& Jun Lv \\ Graduate School, China University of Geosciences, Wuhan 430074, China \\ School of Economics and Management, China University of Geosciences, Wuhan 430074, China
}

\begin{abstract}
The recycle treatment of the municipal solid wastes (MSW) has become an integrated part of the development of recycle economy, it not only helps to alleviate the ecological environment problems brought by a large number of the MSW in the fast development of urbanization in China, but also can obtain urban economic development and some resources which are necessary to residents' life through various kinds of recycle treatment measures. Based on the urbanization development and current situation of the MSW's disposal in China, it have analysed the developing prospect of the recycle treatment industry of the MSW in China, and have structured the social supporting system with three parties' participation which include government, enterprises and residents, so as to promote the development of recycle treatment industry of the MSW in China.
\end{abstract}

Keywords: Urbanization, MSW, Recycle treatment

\section{Introduction}

Since 1990s, in the western developed countries, positive practice of recycle economy has already become the mainstream of economic development, and has obtained remarkable achievement. The trend of the world recycle economy's development embodies mainly in the following aspects such as consummating the legislation of recycle economy gradually, perfecting economic policy of recycle economy and enterprises' active practice of carrying on recycle economy,etc..However, compared with the gradually perfecting situation of foreign recycle economy' development, the development of China's recycle economy still remains in the starting stage of exploring, the scope of recycle economy's development needs expanding badly, the depth demands urgent improvement. For that, this article analyses the developing prospect of the recycle treatment industry of the MSW in China, and structures the social supporting system with three parties' participation which include governments, enterprises and residents, so as to promote the development of recycle treatment industry of the MSW in China.

\section{Urbanization development and current situation of the MSW's disposal in China}

The cities' development can create and gather enormous material wealth and spiritual wealth for a nation. The statistics shows that in China, more than $50 \%$ of the industrial output, more than $70 \%$ of gross national product, nearly $80 \%$ of state finance and tax revenue and $85 \%$ of the third industry 's added value evolves from cities, more than $90 \%$ of the high education and scientific research strength centres in cities(Wang \& Luo, 2005). The city economy's gathering and inducing effects have become obvious day by day. Since the reform and opening-up, China's economy has been keeping fast and continuous development, therefore quickening the urbanization process. During the period of $1995-2005$, China's average annual growth rate of urbanization is $0.8 \%$, after the positive financial policy was implemented in 1998, the speed of urbanization was obviously accelerated. Fig. 1 shows the urbanization rate of China during 1995-2005. it is not difficult for us to predict that the urbanization rate of China will still keep fast growth Within period in the future .

Fast development of urbanization, on the one hand, can give full play to the advantage of the urban gathering, promote the constant development in many aspects like social economy, culture and so on. But on the other hand, cities have a large amount of people who have consumed a large number of materials and resources in the relatively narrow and small space, therefore a large number of the MSW have been produced and have gone beyond cities' self-purifying ability, as a result of that, a series problems of urban ecological environment have been brought out. So, during the process of quickenning the development of urbanization, we should give full play to the advantage of the urbanization in economic and social development, and try to strengthen the construction of environmental-friendly city simultaneously, so as to make the urban economy develop faster and make the urban environmental construction more graceful.

In 2004, the volume of the MSW collected and transported in China reached 155 million tons, and there were 
altogether 559 MSW disposed factories, among which, there were 444 landfill yards, 61 factories of piling, 54 power plants generating electricity through burning, innoxious handling capacity was only 80 million tons, the innoxious handling rate was about $52.1 \%(2005)$. In those western developed countries which are pursueing recycle economy actively, the treatment of the MSW is being developed towards reduction, recycle treatment and innoxious, and remarkable achievement has been obtained. Take Germany as an example, family offal's circulation utilization ratio has already been up to $49 \%$ in 2000 , among them, the rate of recovery of packing bottle of glass was $82 \%$, the rate of recovery of the paper packing article was $77 \%$. Therefore, we can draw a conclusion that the level and ability of the MSW disposal in China are very limited, and they demand urgent promotion.

\section{The prospects of China's development in the recycle treatment industries}

The outline of the Eleventh Five-year plan for national economic and social development in the People's Republic of China, which was passed at the fourth meeting of The Tenth National People's Congress, brings forward clearly that we will build a national economic system with low input and high output, low consumption and less discharge, the ability to circulate and sustain, and build a resource-conserving, environment-friendly society", at the same time, it has proposed a series of measures to develop recycleing economy, this has fully reflected China's hope and resolution to change the economic growth mode and develop the recycling economy.

It is the important component of China's development in recycle economy to promote the recycle treatment of the MSW. To realize the industrialization of recycle treatment of the MSW, it actually means following the theory of recycling economy in practice. It is necessary to fully excavate the economic benefit of recycle treatment of the MSW while guaranteeing its environmental and social benefit; and it is also important to constantly improve the recycle treatment rate of the MSW While guaranteeing the innoxious disposal and reduction of the MSW. Finally the recycle treatment level of the MSW will be improved, and therefore promoting the development of recycling economy.

"Rubbish is resources misplaced ", this statement has already got more and more approval. In the western developed countries, $45 \%$ of the output of steel and iron is produced with abolished steel and iron material, $35 \%$ of the output of copper, $22 \%$ of the output of plumbum , $30 \%$ of the output of zinc , $35 \%$ of the output of paper are all produced by utilizing old and useless material. According to statistics, recycling 1 ton reproducible resources means reducing 4 tons rubbish handling capacity; recycling 1 ton abolished steel and iron can avoid exploiting 20 tons various kinds of ore as well as economize 1.2 tons standard coal for steel and iron-making; The waste paper can produce the good paper, thus economizing the timber; The waste plastics can produce the plastic products, so that the petroleum can be economized. (Luo, 2002)Carrying on recycle treatment to the reproducible material in the MSW is undoubtedly an important way to build a "conservation-oriented society" and develop recycle economy.

According to international trend and domestic practice, reclaiming to reuse, burning to generate electricity and compost are the three most common recycle treatment measures of the MSW. For a long time, the main way of treating the MSW of China is landfill, other ways of recycle treatment are slowly developed. In 2004, there are 444 hygiene landfill yards in China which deal with $68,889,000$ tons MSW altogether, taking $85.2 \%$ of all rubbish handling capacity. Landfill causes problems such as wasting land resources, not dealing with the MSW thoroughly, being apt to lead the secondary pollution, and so on. Moreover, through the way of landfill, some useful material among the MSW might be buried. Therefore, comparatively speaking, though landfill is an simple and easy way to deal with the MSW, and its cost of short-term investment is relatively low, in the long run, recycle treatment measures like reclaiming to reuse, burning to generate electricity and compost can reduce the pollution caused by the MSW to a larger extent, at the same time, they can obtain some resources which satisfy city development and residents' living requirements.

Reclaiming to reuse the MSW include the procedures such as collecting, processing, selling and reusing the useful material in the rubbish. With the improvement of economic development and resident's consuming level, there are more and more useful materials (take paper, metal and plastics as representatives) in the MSW. Therefore, through reclaiming to reuse, renewable resources can be obtained from the MSW, and higher economic benefits can be produced. In the developed countries, the ratio of reclaiming to reuse the waste steel and iron, waste copper and waste rubbers is all up to $90 \%$ while the ratio of reclaiming to reuse the waste steel and iron in China is only $45 \%$, the ratio of reclaiming to reuse waste copper, waste rubbers is $30 \%$ and $40 \%$ respectively(2005). According to the incomplete statistics, every year in China, there are nearly 5 million tons of waste steel and iron, 200,000 tons waste non-ferrous metals, 14 million tons of waste paper, and there is also a large amount of waste plastics and waste glass which is reclaimed and reused incompletely. As a result, the yearly value of the regenerated resources, which could have been recycled if they had been reclaimed, is up to 300 to 350 hundred million yuan. Therefore, China's recycle market of the MSW has a bright future, the potentiality of regenerated resources industry's development is enormous. 
The outstanding advantage of generating electricity through burning the MSW is that it can deal with the MSW more completely, and moreover, it can offer electric energy and heat energy without taking up much land resources. However,generating electricity through burning the MSW expect relatively much combustible content in the rubbish, and it may cause secondary pollution.Besides, the enormous investment in facilities is another problem. At present, the quantity of the power plants generating electricity through burning the MSW, which has already been built up and come into operation in China, is relatively less. But in some large and medium cities and coastal cities, considering factors such as the scarcity of land resources, the short supply in electricity and the great improvement in relevant technology, generating electricity through burning the MSW has already been classified as one of the important ways to dispose the MSW.

The compost refers to the biochemical course of utilizing the technology of fermenting microorganism to promote the degradative organic matter to transform to the steady humus. It is suitable for the MSW with the higher content of organic matter, for instance coal ash, kitchen remains, fallen leaf,etc.. After the MSW is converted to compost, they can be directly used as the fertilizer in agricultural production. Compared with chemistry fertilizer, the MSW compost has relatively low harm whereas its efficiency is relatively bad. The materials like metal, glass and plastics in the MSW will reduce the quality of the compost products so that the sale of the compost products will be influenced. Therefore, the compost technology has relatively higher requirements to the MSW's classification and selection. In recent years, some cities have actively developed the MSW's classification and selection, popularized the mechanized operation, improved the technology of compost, as a result of that, compost has got new development and more and more close attention.

To sum up, compared with fast-developing process of urbanization, the recycle treatment of the MSW in China lags behind seriously, which makes a large number of useful resources in the MSW unable to get effective use in time, and makes them become the trouble of destroying the urban ecological environment instead.Therefore, accelerating the speed of changing the situation that landfill is still considered as the leading status in all the ways of disposing the MSW in China, developing some measures of recycle treatment such as reclaiming to reuse, generating electricity through burning the MSW, compost ,etc. according to the practical circumstances, newly building or reconstructing the facilities for recycle treatment of the MSW, advancing the recycle treatment industry of the MSW towards the directions of scaling, intensifing and modernization, should become the most important thing of the MSW management of our country at present and even in in a very long time of the future.

\section{Discussions on tactics which promote the development of recycle treatment industry of the MSW in China}

As the important component of recycle economy's development, it is a systematic engineering to promote the development of recycle treatment industry of the MSW, it is necessary to carry on the policy planing from the following aspects like governments, enterprises and urbanites progressively, the social supporting system for recycle treatment of the MSW should be set up, and a series of measures such as takeing government's relevant regulation and policy as the leading factor, and taking enterprises' extensive participation and residents' active cooperation as support, should be adopted so as to promote and realize the industrialized development of recycle treatment of the MSW.

\subsection{Governments accelerate the speed of making the relevant regulation and policy, building a good outside environment for development}

The government's relevant departments should play a leading role consciously, improve administrative efficiency, establish the guidelines of keeping harmonious relations between man and nature, establish the strategy for the development of

resource-conserving and environment-friendly cities, strengthen the comprehensive utilization and conservation of resources, especially the utilization of the re-useable material in the MSW, do well the comprehensive tackling and programming of cities' environment, push the all-round development of recycle treatment industry of the MSW.

(1) Set up sound laws and regulations, standardize the relevant behavior subject of recycle treatment of the MSW. In the current laws and regulations system, those closely related to recycle treatment of the MSW mainly include, The Management Rule of Urban Appearance and Environmental Sanitation which was released by The State Council in 1992, the Management Method of the MSW which was released by the Ministry of Construction in 1993), the Prevention and Tackling Law of the Environmental Pollution by the MSW in P.R.C which was released by the National People's Congress in 1995 and revised in 2004. Besides, each local government has made some local rules of law according to the practical development. However, many problems still exist in the formulation and execution of these laws and rules. First, some of the laws and rules are so outmoded that they are unable to solve all sorts of new problems appearing in the fast development of urbanization. Second, it is difficult to restrict and standardize the relevant subjects' behavior because of the incomplet execution of laws and rules. Third, some fuzzy concepts in the 
laws and rules make the right, responsibility and profit of the behavior subjects not well-defined. Therefore, according to the new trend of the development of the MSW and the defect existing in laws and rules, we should gradually revise and perfect the relevant clauses of the laws and rules progressively, strengthen the lenforcement of the existing laws and rules, and insist on the principle of "there must be laws to go by ,the laws must be observed and strictly enforced, and law-breakers must be prosecuted ", so as to give full play to the function of the laws and rules.

(2) Make the perfect policy system, encourage the multi-investing subjects to participate in the recycle treatment of the MSW. The essential prerequisite to realize the recycle treatment of the MSW is to make it profitable. The cost to revert the renewable material in the MSW into the available resource is relatively high, which makes the economy of the relevant products relatively bad, and makes the market competitiveness not strong. In addition, because the construction and operation cost of the recycle treatment facilities of the MSW is usually relatively high, and funds that government's financial budget could allocate to the sanitation department and urban construction department are limited, as a result of that,it is difficult to fulfill the construction and maintenance of some recycle treatment facilities. Therefore, in virtue of the propping up from government's perfect policy system, we must adopt economic means, such as finance, tax revenue ,etc., extensively absorb the foreign and private capitals to enter the recycle treatment industry of the MSW, optimize the mechanism of market competition, foster the good serving market, encourage scientific and technical innovation, create the market environment of equity,justness and open, lead the recycle treatment industry of the MSW to develop in a healthy and orderly manner.

\subsection{Enterprises' extensive participation make them become the mainstay of recycle treatment of the MSW}

The enterprises of environmental protection type are concrete implementers of recycle treatment of the MSW, they should become the mainstay of the recycle treatment industry of the MSW. According to the request for the development of recycle economy, enterprises can carry on activities of producing and managing from transverse and vertical angles, and actively launch the recycle treatment of the MSW.

From the transverse angle, enterprises of environmental protection type should enter the ecological industrial park and new high-tech industry garden initiatively, fully utilize the characteristic of closed circulation of substances in industrial system, through the transverse coupling and resource-sharing between different enterprises or industrial procedures, find enterprises' upriver suppliers of raw materials accurately, and find the wastes analyst of the low reaches at the same time. According to the interdependence relation between different enterprises, form relevant industry clusters, set up food chain and food network of the industry ecosystem in the garden, so as to achieve the goal to turn the negative benefit of pollution into positive benefit of resources (2005).

From the vertical angle, enterprises should pursue cleaner production and green operation from the inside, carry out the thought of recycle economy in all respects of the production and management and bring them into effect, launch from the respects like product design, technological progress and scientific management and so on, economize resources and protect the environment to the maximum extent. In addition, enterprises should also use renewable raw materials and products to carry on the production and management as much as possible, take the initiative to implement the system of producers taking responsibility, actively do a good job of reclaiming to reuse of products, form the material circulation in which enterprises themselves are taken as the centre to enclose.

\subsection{Residents' active cooperate establishes the mass foundation of recycle treatment of the MSW}

The urban residents' daily life and relevant activities are closely linked with the MSW. Firstly, the MSW directly root in the urban residents' daily life and relevant serving activities. Secondly, the way that residents handle the MSW will influence the methods and efficiency of the treatment to the MSW and vice versa. Therefore, it is reasonable for the urban residents to be obligated to actively participate in the activities like collecting and classifying the MSW. Meanwhile, because of the external diseconomy of the MSW's disposal, urban residents are unwilling to take the initiative to undertake these duties. All in all, on the one hand, propagating education and moral remonstration to the urban residents should be launched, and the serious harm caused by the MSW should be expound with the help of the public opinion and news media, the important meaning of categorised collection of the MSW should be propagated, and the urban residents should be encouraged to actively participate in the categorised collection of the MSW. On the other hand, the government organs could adopt the methods of legislating, administration, collecting charges and taxes, carry on necessary restriction or encouragement to the urban residents' behaviors of disposing the MSW. As a result of that, the activity of abandoning the MSW without classification could be limited, and the categorised collection and reclaiming to resue the MSW could be promoted (Zhang, 2004).

\section{Conclusion}

Realizing the recycle treatment of the MSW in China, on the one hand,can help to solve the urban ecological environment problems that the MSW causes, and have very strong urgencies, on the other hand, can help to obtain 
some resources necessary for city development and residents' life and make the market development's prospect brighter. However, it is a long-term evolution for the development of the recycle treatment of the MSW, it relies on the support of various aspects, such as politics, economy, culture, science and technology, etc.. Therefore, we should take the actual development in different places into consideration, adjust measures to local conditions, choose the suitable ways of recycle treatment and actively popularize them, perfect the relevant supporting system, so that the industrialized development of recycle treatment of the MSW in China could be realized in the end.

\section{References}

Wang, YanChun \& Luo, QiShan. (2005) Analysis on Problems of Urbanization in China Under the Goal of Building a Moderately Properous Society in All Respects. The Exploration on Economic Problems, (6).

Statistics Bureau of the People's Republic of China. (2005). Chinese Statistical Yearbook of 2005. Beijing: Chinese Statistics Press,.

Luo, Lan. (2002).The Rubbish is Resources Misplaced - --The survey of issues on the recycling of Wastes in China. People's Daily (overseas edition), 07-31.

(2005). The Centre Promoting the International Enviromental Protection Industry in Zhongguancun. The International Trend of recycle economy and the practice in China. Beijing: People's Press.

Zhang, Yue. (2004). Economics of Management of the MSW's Reduction. Beijing: Chemical Industry Press.

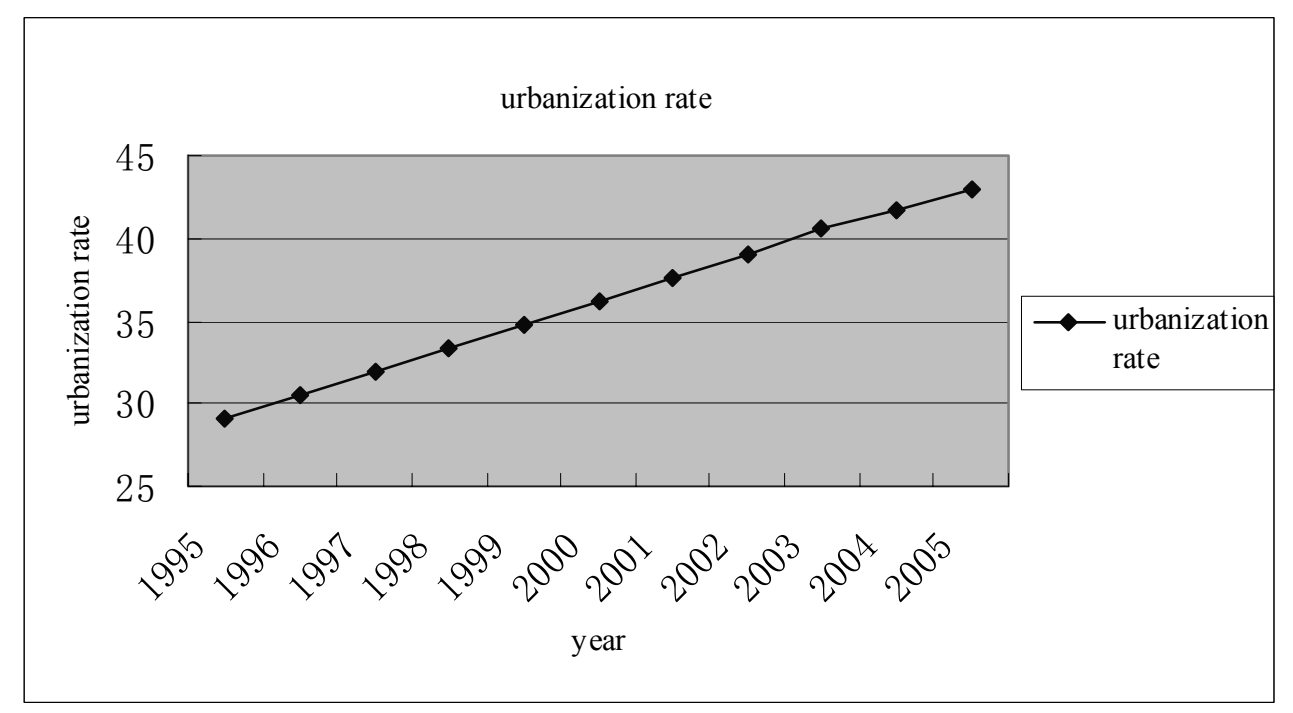

Figure 1. Urbanization rate of China during 1995-2005 